\title{
On the Steady States of Finitely Many Chemical Cells
}

\author{
By J. Bigge and E. Bohl
}

\begin{abstract}
Mathematical models of the form (1) and (2) for diffusion-reaction phenomena are discussed. The occurrence of bifurcation points in the discrete case (2) is explained via a simple two-dimensional model.
\end{abstract}

0. Introduction. Steady state models for transport phenomena in chemistry and biology are usually of the general form

$$
\left(p y^{\prime}\right)^{\prime}-(v y)^{\prime}=g(y) \text { on }[0,1] .
$$

The function $p=p(s)$ describes the diffusion coefficient and $v=v(s)$ the velocity of an underlying flow bringing about convective mass transport. The function $g$ stands for the generation part of the process. We need boundary constraints to complete the model. Normally they have the form of two-point linear conditions.

A straightforward numerical model for (1) reads as follows: let $N \in \mathbf{N}, h=$ $(N+1)^{-1}, s_{j}=j h(j=0, \ldots, N+1)$, and approximate (1) by

$$
\begin{aligned}
& h^{-2}\left(\left(p\left(s_{j}-0.5 h\right)+h v\left(s_{j-1}\right)\right) y\left(s_{j-1}\right)\right. \\
& \left.\quad-\left(p\left(s_{j}-0.5 h\right)+p\left(s_{j}+0.5 h\right)+h v\left(s_{j}\right)\right) y\left(s_{j}\right)+p\left(s_{j}+0.5 h\right) y\left(s_{j+1}\right)\right) \\
& \quad=g\left(y\left(s_{j}\right)\right) \quad(j=1, \ldots, N) .
\end{aligned}
$$

This amounts to using central differencing on $\left(p y^{\prime}\right)^{\prime}$ and backward differencing on $(v y)^{\prime}$.

Many recent papers [1], [4], [6]-[9], [12], [13] report differences between the bifurcation diagrams of (1) and of (2) (with respect to some control parameter). New bifurcation points occur and even complete extra solution branches show up for (2). All of these "discrete phenomena" disappear locally only in the limit $h \rightarrow 0$.

In this paper, we want to stress the point that (2) may be interpreted as a direct mathematical model for a collection of $N$ separated cells communicating with each other through membranes which allow for diffusive or convective mass transport. Each of these cells exhibits a chemical reaction generating (or consuming) a substrate $S$. Hence, the mass transport is completely confined to the membranes, whereas the reaction part is strictly left to the cells. This type of system has also been studied in [2], [10], [11]. It has more stable steady states than a system which is described by the continuous model (1) since parts of the extra solution branches to

Received August 8, 1982; revised June 21, 1984.

1980 Mathematics Subject Classification. Primary 65L10, 92A05, 92A40, 80A30, 80A20.

1985 American Mathematical Society $0025-5718 / 85 \$ 1.00+\$ .25$ per page 
(2) mentioned above are stable. For the case $p \equiv 1, v \equiv 0$ and an inhibited Michaelis-Menton process in the cells, this has been studied in [7b], [10]. If $p \equiv 1$ and $v \equiv v_{0} \gg 1$, special phenomena for (2) have been obtained [5] (see also [6]) under the assumption of an exothermic first-order reaction yielding the function $g$ given in Section 2. However, it is pointed out that the phenomena are independent of this special function $g$. They are rather due to a qualitative behavior of $g$ described in [5], [6] and occur for numerous further nonlinearities including the Michaelis-Menton process mentioned above.

The organization of this paper is as follows: In Section 1 we describe the finite cell model with the special reaction term $g$ given in Section 2. Section 3 comments on numerical experiments. A closed branch of unsymmetric solutions bifurcates from the main branch of symmetric solutions. Small perturbations on the diffusion mechanism resolves the two bifurcation points to form two separate branches. To explain these phenomena a simpler mathematical model is set up in Section 4. It is based on the numerical results of Section 3, which say that only the cells at both ends of the system (cell number 1 and $N$ ) undergo substantial changes along the branch of unsymmetric solutions. All the other cells (number 2 to $N-1$ ) operate on an almost constant level. This observation breaks the discussion down to only two uncoupled equations. The discussion of this system in Section 4 explains the numerical findings of Section 3.

The second author extends his thanks to Prof. Dr. J. Descloux for an inspiring discussion which initiated this paper.

1. The Cell Model. Consider a collection of $N$ cells $Z_{j}(j=1, \ldots, N)$ as shown in Figure 1. In each cell the same kind of chemical reaction takes place transforming a substrate $S$ into a product. Each cell is assumed to be well stirred at any moment and to function independently of all other cells. For any $j=1, \ldots, N-1$, the cell $Z_{j}$ communicates with the cell $Z_{j+1}$ through a membrane $M_{j+1}$ allowing diffusive or convective mass transport. No reaction occurs on the way through the membrane. We consider one-dimensional mass transport only along the horizontal axis. Hence, for setting up a mathematical model, Figure 1, b) is appropriate. It indicates the length $h_{j}$ of the cell $Z_{j}$ and the width $k_{j}$ of the membrane $M_{j}$ between $Z_{j-1}$ and $Z_{j}$.

Let us assume that the total flux through the membrane $M_{j}$ is in one direction only: either from $Z_{j-1}$ to $Z_{j}$ or from $Z_{j}$ to $Z_{j-1}$. The quantity $J_{j}$ is defined as follows:

$$
J_{j}=\left\{\begin{array}{l}
\text { total flux per time unit through } M_{j}, \text { if } \\
\text { the direction of the flux is from } Z_{j-1} \text { into } Z_{j} ; \\
\text { the negative of the total flux per time unit } \\
\text { through } M_{j} \text { otherwise. }
\end{array}\right.
$$

\begin{tabular}{l|l|l|l|l}
\hline$z_{j-1}$ & $M_{j}$ & $z_{j}$ & $M_{j+1}$ & $z_{j+1}$ \\
\hline
\end{tabular}

b)

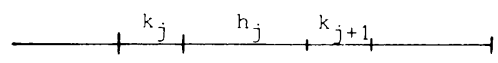

Figure 1 
We denote by $c_{j}=c_{j}(t)$ the concentration of the substrate $S$ in the cell $Z_{j}$ at the time $t$. Then $\Delta t^{-1}\left(c_{j}(t+\Delta t)-c_{j}(t)\right)$ describes the (discrete) rate of change of the concentration of $S$ in $Z_{j}$ per time unit. The basic mass balance equation for $S$ in $Z_{j}$ reads

$$
\Delta t^{-1}\left(c_{j}(t+\Delta t)-c_{j}(t)\right)=J_{j}-J_{j+1}-h_{j} g\left(c_{j}\right),
$$

where $-g\left(c_{j}\right)$ describes the total consumption of $S$ per volume unit and time unit in the cell $Z_{j}$. The steady state equation is

$$
J_{j}-J_{j+1}=h_{j} g\left(c_{j}\right) .
$$

We consider two types of mass transport: diffusive flux

$$
J_{j}^{d}=-D_{j} k_{j}^{-1}\left(c_{j}-c_{j-1}\right)
$$

or convective flux (due to a flow of constant velocity $\lambda_{j}$ from $Z_{j-1}$ into $Z_{j}$ through $\left.M_{j}\right)$

$$
J_{j}^{c}=\lambda_{j} c_{j-1} .
$$

The quantity $D_{j}$ in (5) stands for the diffusion coefficient of the membrane $M_{j}$. In general, the total flux is the sum of $J_{j}^{d}$ and $J_{j}^{c}$, hence

$$
J_{j}=J_{j}^{d}+J_{j}^{c} \text {. }
$$

Now, (4)-(7) yield the basic discrete steady state model:

$$
\begin{aligned}
-D_{j} \frac{c_{j}-c_{j-1}}{k_{j}}+D_{j+1} \frac{c_{j+1}-c_{j}}{k_{j+1}}+\lambda_{j} c_{j-1}-\lambda_{j+1} c_{j}=h_{j} g\left(c_{j}\right) & \\
& j=1, \ldots, N .
\end{aligned}
$$

The model consists of $N$ equations for $N+2$ unknowns $c_{0}, c_{j}(j=1, \ldots, N), c_{N+1}$. The physical interpretation of $c_{0}$ and $c_{N+1}$ is as yet undefined. Indeed, $c_{0}$ and $c_{N+1}$ are given via boundary constraints. In the simplest case $c_{0}$ and $c_{N+1}$ are concentrations of the substrate $S$ being fed to the system from either end. If we want zero diffusive flux on both ends we find

$$
c_{0}=c_{1}, \quad c_{N+1}=c_{N}
$$

from (5). In general, we have to add two (normally linear) equations to the system (8) for the unknowns $c_{0}$ and $c_{N+1}$.

If the total consumption of $S$ per volume unit and time unit depends on the cell $Z_{j}$, then we have to consider $g$ in (8) to depend on $j: g\left(c_{j}\right)=g_{j}\left(c_{j}\right)$.

If we let $k_{j}=h_{j}=h$ for $j=1, \ldots, N$, our model simplifies to

$$
\begin{aligned}
h^{-2}\left(\left(D_{j}+h \lambda_{j}\right) c_{j-1}-\left(D_{j}+D_{j+1}+h \lambda_{j+1}\right) c_{j}+D_{j+1} c_{j+1}\right)= & g\left(c_{j}\right), \\
& j=1, \ldots, N .
\end{aligned}
$$

It is now obvious that the numerical model (2) to the boundary value problem (1) discussed in the introduction is a special case of (8). Here, $h=(N+1)^{-1}$ and $h \rightarrow 0$ which describes "convergence of (2) to the boundary value problem (1)" means for our discrete model that the length of the cells and the width of the membranes go to zero where, at the same time, the number of cells tends to infinity.

Let us return to the general case of the system (8) supplemented by two linear equations for $c_{0}$ and $c_{N+1}$. The left-hand side describes an $(N+2 \times N+2)$-matrix 
( $-A$ ) and the right-hand side defines a diagonal mapping $G$ of $\mathbf{R}^{N+2}$ into itself given by

$$
\begin{array}{ll}
(G c)_{j}=g\left(c_{j}\right) & (j=1, \ldots, N), \\
(G c)_{j}=\gamma_{j} & (j=0, N+1),
\end{array}
$$

where $\gamma_{j}$ denotes a constant given by the boundary constraints. Note that the $j$ th equation of (8) is multiplied by $h_{j}^{-1}(j=1, \ldots, N)$. With this notation, the system (8) may be written as

$$
A c+G c=\theta
$$

where $c=\left(c_{0}, \ldots, c_{N+1}\right) \in \mathbf{R}^{N+2}$.

2. First-Order Exothermic Reaction Term. To perform our numerical experiments we need a more specific situation. As to the boundary constraints we assume Dirichlet conditions

$$
c_{N+1}=c_{0}>0 \quad\left(c_{0}=\text { prescribed }\right) .
$$

This means that the concentration of the substrate $S$ is kept on the constant level $c_{0}$ at both ends of the system.

Next we turn to the function $g$. To this end we consider an exothermic first-order reaction going on in each of the cells $Z_{j}$. This leads to

$$
g\left(c_{j}\right)=k\left(T_{j}\right) c_{j}, \quad k\left(T_{j}\right)=k_{0} \exp \left(-\frac{Q}{R T_{j}}\right),
$$

where $T_{j}$ is the absolute temperature and $k_{0}, Q, R$ are constants [2], [7b]. In many situations a linear relation $T_{j}-T_{0}=\mu_{0}\left(c_{0}-c_{j}\right)$ is satisfied with a constant $\mu_{0}>0$ and the temperature $T_{0}$ on both ends of the system. Following the normal procedure in the literature we introduce the (dimensionless) constants

$$
\beta=\mu_{0} c_{0} T_{0}^{-1}, \quad \gamma=Q R^{-1} T_{0}^{-1}
$$

and the (dimensionless) variable

$$
x_{j}=\beta c_{0}^{-1}\left(c_{0}-c_{j}\right) \quad(j=0, \ldots, N+1) .
$$

The constraint (13) implies

$$
x_{0}=x_{N+1}=0
$$

and the system (12) transforms into

$$
\begin{gathered}
A x=F x+\beta(A \delta-r), \\
(F x)_{0}=(F x)_{N+1}=0, \\
(F x)_{j}=k_{0}\left(\beta-x_{j}\right) \exp \left(-\gamma\left(1+x_{j}\right)^{-1}\right) \quad(j=1, \ldots, N), \\
r=(1,0, \ldots, 0,1) \in \mathbf{R}^{N+2}, \quad \delta=(1,1, \ldots, 1) \in \mathbf{R}^{N+2}
\end{gathered}
$$

(see also [7b]). Note that

$$
(A \delta)_{0}=(A \delta)_{N+1}=1, \quad(A \delta)_{j}=h_{j}^{-1}\left(\lambda_{j+1}-\lambda_{j}\right) \quad(j=1, \ldots, N),
$$

so that $A \delta-r=\theta$ if $\lambda_{j+1}-\lambda_{j}=0(j=1, \ldots, N)$. In this case, the system (16) reduces to

$$
A x=F x \text {. }
$$


3. Numerical Experiments. The basis of the following numerical experiments is the system (17) with

$$
\begin{gathered}
N=9, \quad k_{0}=10^{12}, \quad \beta=1, \\
k_{j}=h_{j}=0.1, \quad D_{j}=1+0.1(j-0.5) d, \quad \lambda_{j}=0 \quad(j=1, \ldots, 10) .
\end{gathered}
$$

Hence, the system reads

$$
\begin{gathered}
x_{0}=0, \\
-(100+10(j-0.5) d) x_{j-1}+(200+20 j d) x_{j} \\
-(100+10(j+0.5) d) x_{j+1} \\
=10^{12}\left(1-x_{j}\right) \exp \left(-\gamma\left(1+x_{j}\right)^{-1}\right), \quad(j=1, \ldots, 9), \\
x_{10}=0,
\end{gathered}
$$

where $d$ is the diffusion control parameter and $\gamma$ is the temperature control parameter (variation of $\gamma$ means variation of $T_{0}$, see [2], [7b]). We mention that $k_{0}=10^{12}$ is chemically realistic (see [2], [7b]).

We begin with symmetric diffusion and put $d=0$, hence $D_{j}=1(j=1, \ldots, 10)$. The resulting bifurcation diagram is given in Figure 2. All solutions are chemically relevant in the sense that they all meet the a priori bounds

$$
0 \leqslant x_{j}(\gamma) \leqslant 1 \quad(j=0, \ldots, 10)
$$

suggested by the transformation (14). Furthermore, the main branch consists of only symmetric solutions

$$
x_{j}(\gamma)=x_{10-j}(\gamma) \quad(j=0, \ldots, 5)
$$

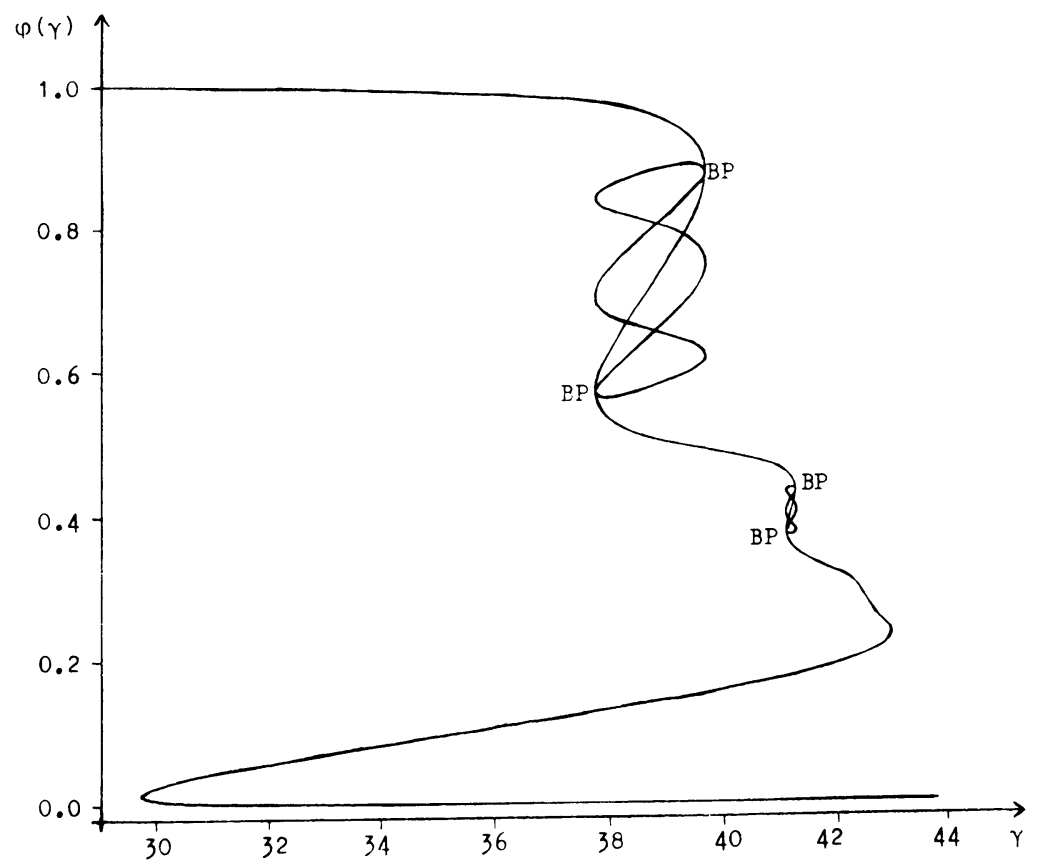

FIGURE 2 
This is no longer true for the bifurcating branches exhibiting the shape of a double-figure-eight, whose crossing points are not bifurcation points (all bifurcation points are marked as BP). It is a closed loop in the space $\mathbf{R}^{9} \times \mathbf{R}$ projected onto the plane $\mathbf{R}^{2}$ via the functional

$$
\gamma \rightarrow \frac{1}{3}\left(2 x_{1}(\gamma)+x_{9}(\gamma)\right)=: \varphi(\gamma)
$$

All branches have stable parts (solid lines, see Figure 3) and unstable parts (broken lines, see Figure 3). Here, stability of $x(\gamma)$ means that the linearization of the system (19) at $x(\gamma)$ has a nonnegative inverse which is equivalent to saying that $x(\gamma)$ is a stable steady state solution of the corresponding dynamical system (3). Note that the double-figure-eight structure shows up twice in Figure 2.
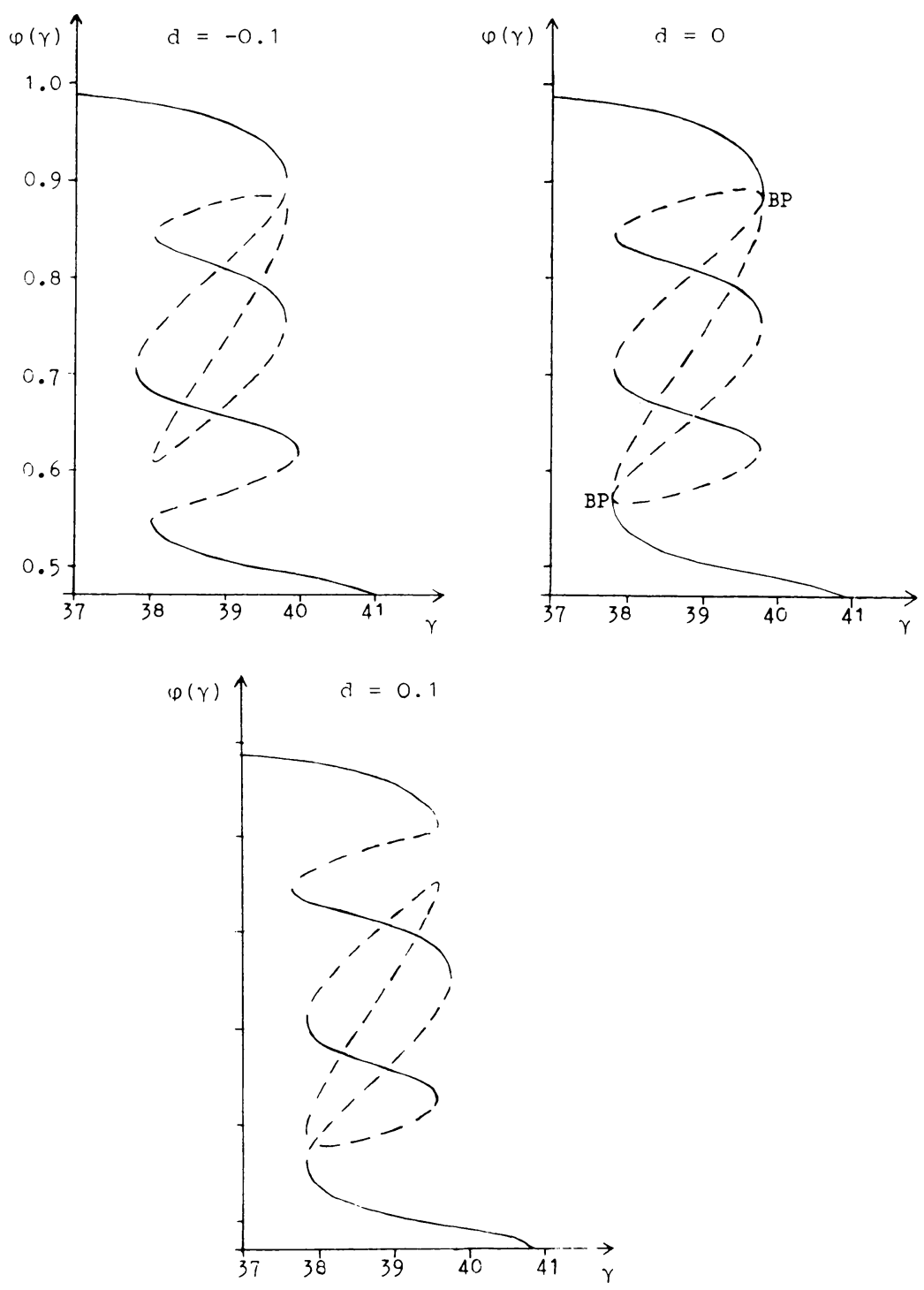

FIGURE 3 
In the spirit of the interpretation of (19) as a model for 9 chemical cells, we find that these cells do not only operate in a symmetric way (see (20)) but they also can work together on nonsymmetric, stable steady states (see also [10] for further examples for this phenomenon). This property of the system (19) is not understandable if we interpret (19) as finite-difference approximation to the continuous model

$$
\begin{gathered}
-x^{\prime \prime}=10^{12}(1-x) \exp \left(-\gamma(1+x)^{-1}\right), \\
x(0)=x(1)=0
\end{gathered}
$$

on the grid $t_{j}=0.1 j(j=0, \ldots, 10)$. It can be shown that (22) admits only symmetric solutions: $x(s)=x(1-s)$. Furthermore, the solution branch satisfying $0 \leqslant x(\gamma, s) \leqslant 1 \quad(s \in[0,1])$ has no bifurcation point (see [3]). As a result, the double-figure-eight of Figure 2 must disappear in the limit $h \rightarrow 0$. This limiting process means in the cell model that the number of cells becomes very large whereas the width of the cells and the intermediate membranes shrink to zero.

Next, we want to study the effect of the "perturbation parameter" $d$ in (19) on the bifurcation phenomenon in Figure 2. We put $d= \pm 0.1$ which amounts to a nonconstant diffusion activity of the membranes: $D_{j}=1+0.1(j-0.5) d(j=$ $1, \ldots, 10)$. The result is Figure 3, which is a close up of the upper part of Figure 2 in the perturbed situation. Both bifurcation points are resolved. A new main branch and a free figure-eight-branch is created out of the original situation shown in Figure 2. The two separated branches have stable (solid lines) and unstable (broken lines) parts. The main branch shows an additional hysteresis loop which comes from unsymmetric parts of the unperturbed picture. For example, the point

$$
\gamma=39.2, \quad \varphi(\gamma)=0.7748 \ldots
$$

on the free figure-eight for $d=0.1$ deforms for $d \rightarrow 0$ into

$$
\gamma=39.2, \quad \varphi(\gamma)=0.7674 \ldots
$$

which is on the symmetric (main) branch of Figure 2. The continuation process $d=10^{-j}$ for some $j \in \mathbf{N}$ is shown in Table 1 . A similar continuation process (see Table 2) deforms the point

$$
\gamma=38.2, \quad \varphi(\gamma)=0.756 \ldots
$$

on the free figure-eight into the point

$$
\gamma=38.2, \quad \varphi(\gamma)=0.758 \ldots
$$

which corresponds to an unsymmetric solution (see Table 2). This shows that, with the resolution of the bifurcation point, parts of the symmetric branch and of the unsymmetric branch in Figure 2 come together to form the new main branch of Figure 3. 
TABLE $1 . d=10^{-j}, \gamma=39.2$

\begin{tabular}{l|lll}
$j$ & $x_{1}(\gamma)$ & $x_{9}(\gamma)$ & $\varphi(\gamma)$ \\
\hline 1 & 0.7677 & 0.78 & 0.7748 \\
2 & 0.7674 & 0.7696 & 0.7682 \\
3 & 0.76747 & 0.7676 & 0.7675 \\
4 & 0.76747 & 0.76749 & 0.7674 \\
5 & 0.767476 & 0.767479 & 0.7674 \\
6 & 0.767476 & 0.767477 & 0.7674 \\
7 & 0.767476 & 0.767476 & 0.7674 \\
$\infty$ & $0.767476 .$. & $0.767476 .$. & 0.7674
\end{tabular}

TABLE 2. $d=10^{-j}, \gamma=38.2$

\begin{tabular}{l|lll}
$j$ & $x_{1}(\gamma)$ & $x_{9}(\gamma)$ & $\varphi(\gamma)$ \\
\hline 1 & 0.6476 & 0.9735 & 0.7562 \\
2 & 0.6487 & 0.9760 & 0.7578 \\
3 & 0.6489 & 0.9763 & 0.7580 \\
$\infty$ & $0.6489 .$. & $0.9763 .$. & 0.7580
\end{tabular}

4. Understanding the Results of Section 3. In this section we try to understand the phenomena described in Section 3. A glance at the figures of Tables 3 and 4 reveals that for $\varphi(\gamma) \geqslant 0.56$, the components $x_{j}(\gamma)(j=2, \ldots, 8)$ are almost constant. Since $x_{0}(\gamma)=x_{10}(\gamma)=0$, we find that only $\gamma, x_{1}(\gamma), x_{9}(\gamma)$ show substantial variations. Note that in the discrete cell model the cells in the middle show almost no concentration $c_{j}$ (see (14), recall $\beta=1$ by (18a) and $x_{j}(\gamma) \approx 0.99$ for $\left.j=2, \ldots, 8\right)$ at any symmetric or unsymmetric state.

TABLE 3. $d=0$, symmetric solutions (see (20)).

\begin{tabular}{l|lll}
$\gamma$ & $x_{1}(\gamma)$ & $x_{2}(\gamma)$ & $x_{3}(\gamma)-x_{5}(\gamma)$ \\
\hline 39.807 & 0.884 & 0.995 & 0.999 \\
39.792 & 0.87 & 0.994 & 0.999 \\
37.895 & 0.6 & 0.993 & 0.999 \\
37.851 & 0.56 & 0.992 & 0.999
\end{tabular}

TABLE 4. $d=0.1$, unsymmetric solutions (main branch).

\begin{tabular}{l|llcccc}
$\gamma$ & $x_{1}(\gamma)$ & $x_{2}(\gamma)$ & $x_{3}(\gamma)-x_{7}(\gamma)$ & $x_{8}(\gamma)$ & $x_{9}(\gamma)$ & $\varphi(\gamma)$ \\
\hline 37.831 & 0.981 & 0.999 & 0.999 & 0.992 & 0.573 & 0.845 \\
38.000 & 0.979 & 0.999 & 0.999 & 0.993 & 0.620 & 0.859 \\
38.573 & 0.97 & 0.999 & 0.999 & 0.992 & 0.694 & 0.878 \\
39.260 & 0.95 & 0.998 & 0.999 & 0.992 & 0.775 & 0.891 \\
39.792 & 0.87 & 0.994 & 0.999 & 0.995 & 0.898 & 0.879
\end{tabular}


STEADY STATES OF FINITELY MANY CHEMICAL CELLS

413

In view of the system (19) our observation means that already the equations for $j=1$ and $j=9$ describe the situation. However, these are two uncoupled equations:

(23a) $(200+20 d) x_{1}=10^{12}\left(1-x_{1}\right) \exp \left(-\gamma\left(1+x_{1}\right)^{-1}\right)+(100+15 d) x_{2}$.

(23b) $(200+180 d) x_{9}=10^{12}\left(1-x_{9}\right) \exp \left(-\gamma\left(1+x_{9}\right)^{-1}\right)+(100+85 d) x_{8}$,

note that $x_{2} \approx x_{8} \approx 0.99$ and $x_{0}=x_{10}=0$. It is easily verified that any solution $\left(\gamma, x_{1}\right)$ of $(23 \mathrm{a})$ and $\left(\gamma, x_{9}\right)$ of $(23 \mathrm{~b})$ (for $\left.x_{2} \approx x_{8} \approx 0.99\right)$ also solves an "explicit system"

$$
\begin{gathered}
\gamma=\bar{f}\left(x_{1}, d\right), \quad \gamma=\bar{g}\left(x_{9}, d\right), \\
\bar{f}(x, 0)=\bar{g}(x, 0) .
\end{gathered}
$$

This system may serve as a model for our full problem (19). The graph of the function $\bar{f}(x, 0)$ is given in Figure 4. Indeed, the general structure of this graph is the essential property to our analysis. Hence, instead of (24a), (24b) we may just as well consider any uncoupled system

$$
\gamma=f(x), \quad \gamma=g(y),
$$

where the graph of $f$ and $g$ is qualitatively given in Figure 4. The simplest example is

$$
f(x)=x^{3}-a x+b, \quad g(y)=y^{3}-c y+e, \quad a, b, c, e>0 .
$$

The set of solutions of (25) is in a qualitative way the same as the corresponding set for (24).

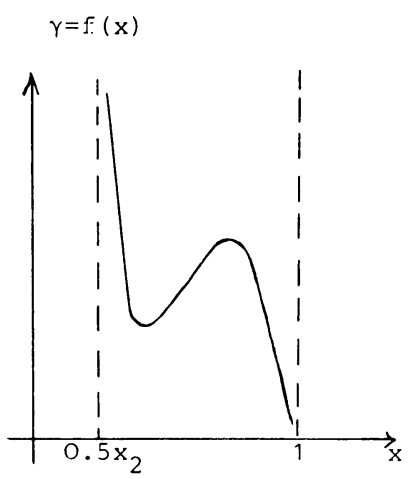

FIGURE Aa

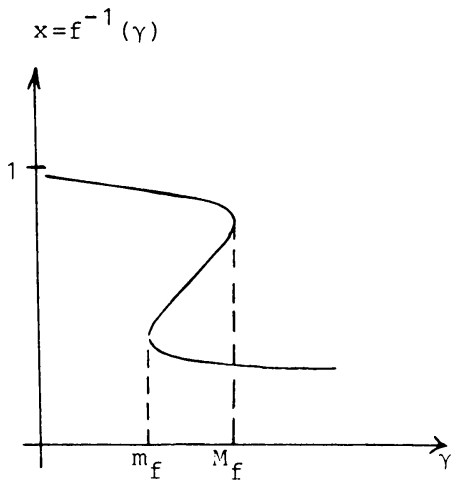

FIGURE Ab

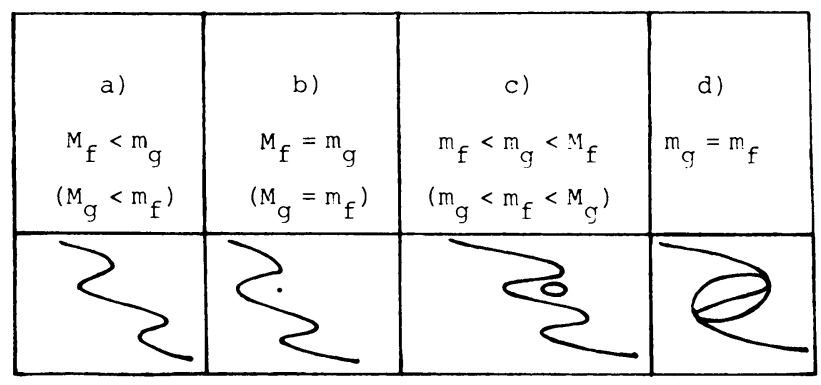

Figure 5 


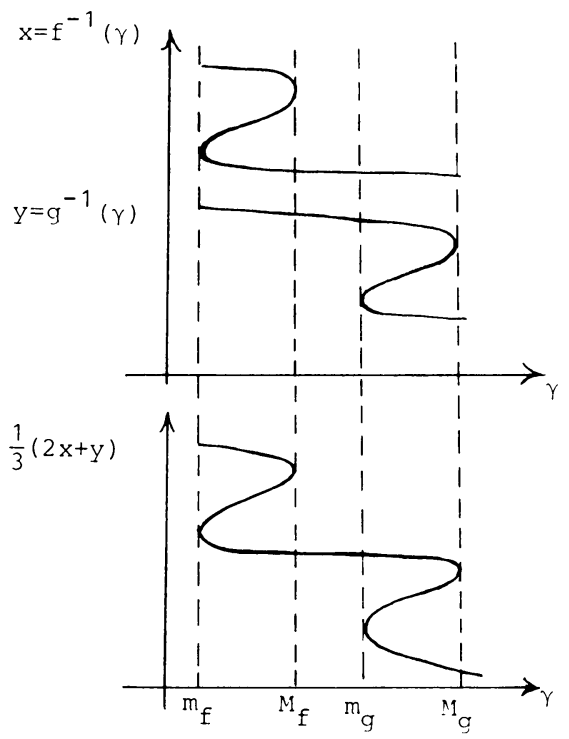

Case (a) of Figure 5

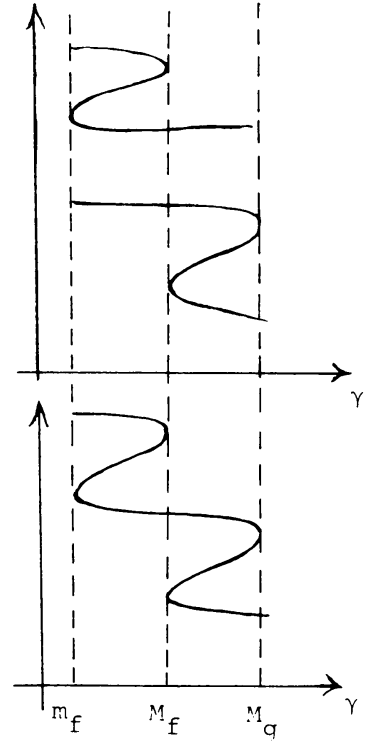

Case (b) of Figure 5

Figure 6

\begin{tabular}{|c|c|}
\hline $\mathrm{d}_{\mathrm{f}}<\mathrm{m}_{\mathrm{g}}, \mathrm{M}_{\mathrm{g}}=\mathrm{M}_{\mathrm{f}}$ & $\left.\mathrm{d}_{2}\right)$ \\
$\left(\mathrm{m}_{\mathrm{f}}=\mathrm{m}_{\mathrm{g}}, \quad \mathrm{M}_{\mathrm{g}}<\mathrm{M}_{\mathrm{f}}\right)$ & \\
\hline & \\
\hline & \\
\hline
\end{tabular}

FIGURE 7

To describe all solutions of (25), we distinguish between the following two cases:

$$
\begin{aligned}
& M_{f}-m_{f}=M_{g}-m_{g}, \\
& M_{f}-m_{f}>M_{g}-m_{g},
\end{aligned}
$$

where $M_{f}, M_{g}$ or $m_{f}, m_{g}$ denote the maximum or the minimum value of $f$ or $g$ as indicated in Figure 4b. Under the hypothesis (27) the structure of the set of all solutions of (25) is given in Figure 5: We have four essentially different sets of solutions depending upon the intersection of the intervals $\left[m_{f}, M_{f}\right],\left[m_{g}, M_{g}\right]$. The set consists of (see Figure 5!):

(a) one branch with some hysteresis loops,

(b) as in (a) with an additional point,

(c) as in (a) with an additional closed branch separated from the one described in (a),

(d) as in (c), but the two branches meet in two bifurcation points. 
Figure 5 represents in a symbolic way the possible combinations of two hysteresis loops as shown in Figure $4 \mathrm{~b}$. The cases (a) and (b) of Figure 5 are given in more detail in Figure 6 as illustrative examples.

Returning to (19) with $d=0$, the upper part of Figure 2 shows the situation (d): the double-figure-eight is the closed branch which meets the branch of symmetric solutions in two bifurcation points. According to our model (see Figure 5), small perturbations must result in the situation c) of Figure 5. Indeed, Figure 3 shows the two separated branches, one of which (the figure-eight) is closed.

We conclude with a brief discussion of the case (28). Here, case (d) of Figure 5 is split into the cases $\left(d_{1}\right),\left(d_{2}\right)$ of Figure 7. $\left(d_{1}\right)$ describes two branches which meet in one bifurcation point. One of these branches is closed. $\left(d_{2}\right)$ results in just one branch which may have a complicated structure of many hysteresis loops.

Fakultät für Mathematik

Universität Konstanz

D-7750 Konstanz 1

West Germany

1. E. Allgower, "On a discretization of $y^{\prime \prime}+\lambda y^{k}=0$ " in Proc. Conf. Roy. Irish Acad.. (J. J. H. Miller, ed.), Academic Press, New York, 1975.

2a. R. ARIS, Mathematical Modelling Techniques, Res. Notes in Math., vol. 24, San Francisco, London, Melbourne, 1968.

2b. R. ARIS, The Mathematical Theory of Diffusion and Reaction in Permeable Catalysts, Vols. I. II. Oxford, 1975.

3. W.- J. BEYN, Lösungszweige nichtlinearer Randwertaufgaben und ihre Approximation mit dem Differenzenverfahren, Habilitationsschrift, University of Konstanz, 1981.

4. W.- J. BEYN \& J. LORENZ, "Spurious solutions for discrete superlinear boundary value problems," Computing, v. 28, 1982, pp. 42-51.

5. J. Bigge, Lösungszweige von diskreten Reaktions-Transport-Modellen, Ph.D-thesis, University of Konstanz, Hartung-Goree-Verl., 1984.

6. J. Bigge \& E. BoHL, "Deformations of the bifurcation diagram due to discretization," Math. Comp. (To appear.)

7a. E. BонL, "On the bifurcation diagram of discrete analogues for ordinary bifurcation problems," Math. Methods Appl. Sci., v. 1, 1979, pp. 566-671.

7b. E. BoHL, Finite Modelle gewöhnlicher Randwertaufgaben, Teubner Studienbücher, Teubner, 1981.

8. E. J. DOEDEL \& W.- J. BEYN, "Stability and multiplicity of solutions to discretizations of nonlinear ordinary differential equations," SIAM J. Sci. Statist. Comput., v. 2, 1981, pp. 107-120.

9. R. GAINES, "Difference equations associated with boundary value problems for second order nonlinear ordinary differential equations," SIAM J. Numer. Anal., v. 11, 1974, pp. 411-434.

10. J. P. KerneveZ, Enzyme Mathematics, Stud. Math. Appl., vol. 10, North-Holland, Amsterdam, 1980.

11. J. P. Kernevez \& D. Thomas, "Numerical analysis of some biochemical systems," Appl. Math. Optim., v. 1, 1975, pp. 222-285.

12. H. O. Peirgen, D. SAUPE \& K. Schmitr, "Nonlinear elliptic boundary value problems versus their finite difference approximations: Numerically irrelevant solutions," J. Reine Angew. Math., v. 322, 1981 , pp. 74-117.

13. H.- O. Peitgen \& K. Schmitr, Positive and Spurious Solutions of Nonlinear Eigenvalue Prohlems, Technical Report No. 42, University of Bremen, 1981. 